\title{
Comparison of Four Physiotherapy Regimens in the Treatment of Long-Term Mechanical Low Back Pain
}

\author{
Olubusola E. JoHNSON ${ }^{1}$, Babatunde O.A. AdEGOKE ${ }^{2}$ and Samuel O. OGUnLADE ${ }^{3}$ \\ ${ }^{1}$ Department of Medical Rehabilitation, Obafemi Awolowo University, Ile-Ife Nigeria \\ ${ }^{2}$ Department of Physiotherapy, University of Ibadan, Ibadan Nigeria \\ ${ }^{3}$ Department of Surgery, University College Hospital, Ibadan Nigeria
}

\begin{abstract}
Objective: This study compared efficacy of combinations of Back Muscles Endurance Exercise (BMEE) and McKenzie Exercise (ME) and McKenzie Back Care Education (MBE) in the management of long term mechanical Low Back Pain (LBP). Subjects and Methods: A single-blind randomized controlled comparative trial was employed. Seventy three participants mean age $45.3 \pm 8.1$ years were recruited for the study but only 53 completed the study. Participants in group A were treated with a combination of BMEE, ME and MBE. Group B: A combination BMEE and MBE. Group C: A combination of ME and MBE. Group D: MBE only. Participants were seen thrice weekly for 8 weeks. They were measured for pain intensity, lumbar flexibility, activities limitation and self esteem. Data were analysed using descriptive and inferential statistics of F-test. Significance was set at 0.05 alpha-level. Results: At the end of the study, the four treatment groups had significant reduction in pain intensity $\mathbf{p}<\mathbf{0 . 0 5}$. Post hoc analysis showed groups $A, B$, and $C$ had significantly greater reduction than $D$, and groups $A$ and $C$ had significantly greater reduction than $B$. Groups $A$, $B$ and $C$ also had significant improvement in activities limitation $p<0.05$. Post hoc analysis showed groups $\mathbf{A}, \mathbf{B}$ and $\mathbf{C}$ had significantly greater improvement than $\mathbf{D}$, and group $\mathbf{B}$ significantly greater improvement than C. Conclusion: Combination physiotherapy regimens proved effective in the management of long- term mechanical LBP. Regimen A is recommended in managing long-term mechanical LBP.
\end{abstract}

Key words: Comparative efficacy, combination physiotherapy regimens, mechanical low back pain

(J Jpn Phys Ther Assoc 13: 9-16, 2010)

Exercises of various types have been used in managing low back pain with varying reported successes ${ }^{1)}$. For instance Nwuga and Nwuga ${ }^{2)}$ studied William's flexion exercise and McKenzie exercise in the treatment of LBP and reported results in favour of McKenzie exercise. McKenzie exercise was designed for management of mechanical low back pain ${ }^{3)}$. Waddell ${ }^{4)}$ submitted that controlled exercises help in restoring function, reducing distress and promoting an earlier return to work in LBP patients. A relationship exists between the occurrence of LBP and decreased low back muscular endurance and endurance exercises that require the use of the back extensors as well as abdominal muscles have been

Received: July 2, 2009

Accepted: February 23, 2010

Correspondence to: Johnson O. E., Department of Medical Rehabilitation, Obafemi Awolowo University, Ile-Ife, Nigeria

e-mail: obnik2001@gmail.com suggested in the management of long-term mechanical $\mathrm{LBP}^{5-11)}$

Low back pain, which is pain in the lumbo sacral region of the back, between the lower margins of the $12^{\text {th }}$ rib and the gluteal folds ${ }^{12)}$, represents a particularly costly sociomedical problem because of the expenditure associated with repeated treatment, and need for social support ${ }^{13}$. It is a leading cause of activities limitation, occurring in similar proportions in all culture ${ }^{14}$. It interferes with quality of life and work performance and is the most common reason for medical consultation ${ }^{15}$. An estimated $70-95 \%$ of any adult population suffers at least one episode of back pain in its lifetime ${ }^{16-18)}$, and $50 \%$ of the cases will recur within 3 months ${ }^{19)}$.

The management of long-term mechanical LBP is an unending task for health service providers ${ }^{20}$ and great efforts have hence been exerted to improve the efficacy of its treatment because of its recurrent or persistent nature ${ }^{21)}$. 
The bulk of the literature on the management of LBP favours active low back treatment programmes that focus on improving aerobic fitness, increasing the strength, endurance and flexibility of the lumbar musculature and ensuring lumbar stability ${ }^{1,9,22)}$. This approach though conservative is active and aims both to restore function quickly and prevent future episodes while avoiding bed rest and narcotic painkillers and surgery, which are regarded as rarely desirable and only necessary in perhaps $10 \%$ of cases ${ }^{1)}$

There is however a great diversity of opinion regarding the nature of LBP, its optimal management ${ }^{23}$ and exercise preferences, although exercise has been found to be the central element in its management ${ }^{9,22,24-26)}$. In view of the foregoing that different exercise techniques have been used in managing LBP with differing results, the following question therefore came to mind, what would be the relative efficacy of combinations of these proven exercise techniques of managing long- term mechanical LBP? This study was therefore aimed at comparing the efficacy of four physiotherapy regimens in the management of long-term mechanical LBP.

\section{Methods}

Seventy three participants with long-term mechanical LBP were recruited for this study but only 53 completed the 8 -week study. They were male and female individuals with history of long- term mechanical LBP of not less than 6 months. They were attending the Physiotherapy Outpatient Department of the University College Hospital Ibadan. Consecutive non-probability sampling technique was used to recruit participants from University College Hospital Ibadan. The fish bowl technique of simple random sampling was used to randomly assign participants into the 4 treatment groups. Participants were allocated into any of the four treatment group consecutively as they reported for the study and according to the alphabet a participant picked from the pool of alphabets A, B, C and D in the bowl; (Fish bowl technique of simple random sampling).

\section{Procedure}

Ethical approval was obtained from the joint University of Ibadan/University College Hospital Institutional Review Committee on Human Subject Research before commencing the study. Procedure of the study was explained to each participant and informed consent was obtained.

The treatment regimens of participants in each of the four groups were:

Group A: A combination of endurance exercise, McKenzie back exercise and McKenzie back care education.

Group B: A combination of McKenzie exercise and
McKenzie back care education

Group C: A combination of endurance exercise and McKenzie back care education.

Group D: McKenzie back care education only (control group).

\section{Interventions}

\section{Pre-treatment Screening}

Participants were referred for physiotherapy by orthopaedists and further screened for their suitability to take part in the study by the researchers using the McKenzie Institute's Lumbar Spine Assessment Format. Information such as age, gender, and onset of back pain were recorded for each participant accordingly.

\section{Instrumentation}

a) Lumbar Flexibility: The modified Schober technique was used to assess lumbar flexbility. With the participant standing erect but relaxed, the researcher identified the top of the sacrum as 0 , by the spinal intersection of a horizontal line joining the dimples of Venus, and points $10 \mathrm{~cm}$ above and $5 \mathrm{~cm}$ below the horizontal line were marked. The participant was then asked to bend forward maximally while the distance between the upper and lower marks was measured in centimetres. This value minus 15 represented lumbar flexibility ${ }^{27)}$.

b) Pain Intensity: The Numeric Pain Scale was used to measure the level of pain being presently experienced by the participants. The researcher explained the scale to the participant, who was asked to identify his /her, present level of pain and the participant's response was then recorded. Mark 10 stands for most severe pain while mark 0 stands for no pain ${ }^{28)}$. Pain intensity was measured as present pain at its worst.

c) Activity Limitation: This was assessed using the Roland Morris Low Back Pain and Disability Questionnaire $\left(\right.$ RMLDQ) ${ }^{29)}$.

d) Self-Esteem: This was assessed using a self-esteem questionnaire $^{30)}$ at week 0 of the study. Participants were reassessed for these four variables at the end of the $4^{\text {th }}$ and $8^{\text {th }}$ week of the study. The researchers were blinded to the raw data collected in order to avoid bias.

\section{Intervention}

Participants were asked to wear a light vest and a pair of shorts which allowed for ease of movement when carrying out the treatment.

Group A Subjects: These participants were treated with endurance exercises, McKenzie low back exercises and McKenzie back care education instructions. The endurance exercise regimen comprised three phases viz: warm up, main exercise and cool down.

Warm up: This lasted 8 minutes 
Endurance Exercise: The endurance exercise training comprised the following exercises which have been used by Douglas ${ }^{31)}$.

\section{Exercise 1}

Type: Pelvic tilt: Exercise for the core spinal stabilizer transversus abdominus muscle

Starting Position: Crook lying

Instruction: Participant pulled in the belly-button downwards and tightened the buttocks

\section{Exercise 2}

Type: Hamstring stretch: Exercise for the hamstrings muscle group

Starting Position: Supine lying with arms by the sides

Instruction: Participant raised each lower limb while keeping the knee straight and supporting the leg with both hands so as to go up as far as it could go. Participant held the leg in this position for few seconds.

\section{Exercise 3}

Type: Exercise of the abdominals: Alternate leg and arm raising in supine position

Starting Position: Supine lying with arms by the sides

Instruction: Participant slowly lifted the left arm and the right leg simultaneously and brought them to meet in the air above the chest. Participant slowly lowered them to the original position and repeated the exercise with the other pair of limbs.

\section{Exercise 4}

Type: Exercise of the spinal extensor muscles: Alternate leg and arm raising in prone position

Starting Position: Prone lying with arms straight in front

Instruction: Participant slowly raised the right arm and the left leg simultaneously. Participant slowly lowered them into original position and repeated the exercise with the other pair of limbs.

Exercise 5

Type: Cycling- Exercise for both legs.

Position: Sitting on the bicycle

Instruction: Participant rode on the cycle ergometer against zero resistance.

\section{Exercise 6}

Type: Brisk Walking-Exercise for the general body.

Instruction: Participant walked as fast as he could without running.

Participants performed each exercise for 4 minutes and exercises were continuous and performed sequentially until all the 6 different exercises have been completed. Participants started out at their individual paces until participant got fatigued doing the exercises or completed exercises. When participants got fatigued they were made to rest and continue the exercise afterward. Exercises were progressed fortnightly by increasing the time for each exercise by one minute.

Cool down: This lasted 5 minutes.

McKenzie Exercises: They involved a series of either extension or flexion exercises ${ }^{32)}$. They were used as appropriate according to the McKenzie protocol ${ }^{3)}$.

Back Care Education: The participant was then taught McKenzie back care education for standing, sitting, lifting and other activities of daily living using illustrations by Odebiyi ${ }^{33)}$. Small handbills describing the back care education instructions were given as a reminder for the subjects. Each participant received treatment thrice weekly on alternate days for 8 consecutive weeks.

\section{Data Analysis}

Data obtained was summarised using descriptive statistics of mean and standard deviation. One-way analysis of variance (ANOVA) was also used to; (i) compare the demographic data and baseline parameters of participants in the 4 groups of study (ii) compare the effects of the 4 treatment regimens on pain intensity, activities limitation, lumbar flexibility and self esteem of participants across the eight weeks of the study. Least significant difference post hoc analysis was used to locate the treatment groups that were significantly different through ANOVA. Significance was set at $0.05 \alpha$-level.

\section{Results}

\section{Participants' profile}

Fifty three participants, comprising 21 males (39.6\%) and 32 females $(60.4 \%)$ of the 73 participants recruited during the course of this study completed the 8 week study. Thus a $27.4 \%$ attrition rate was observed in this study. The overall mean age of subjects with LBP in this study was $45.3 \pm 8.1$ years.

One Way Analysis of Variance (ANOVA) at $\alpha=0.05$ showed that there were no significant differences in the pain intensity, activities limitation, lumbar flexibility and self esteem ( $>0.05$ ) of the participants in the 4 study groups at baseline (Table 1). One can therefore largely attribute the results obtained at different points during the study to the effects of the various interventions.

\section{Across group comparison of mean change of subjects' parameters from week 0 to 4 of the study}

At the end of $4^{\text {th }}$ week of the study results showed that there were significant differences in the pain intensity $(\mathrm{p}<0.05)$ and lumbar flexibility $(\mathrm{p}=0.05)$ but there were no significant differences in activities limitation and self esteem ( $p>0.05$ ) (Table 2). Post hoc analysis showed that participants in group $\mathrm{C}$ had significantly higher reduction in pain intensity than those in group D. Also participants in group A had significantly higher increase in lumbar flexibility than those in the other 3 groups (Table 3 ).

Across group comparison of mean change of subjects' 
Table 1. Comparison of participants' parameters at week 0 of the study using ANOVA

\begin{tabular}{lrrrrrr}
\hline $\mathrm{N}=53$ & Grp. A & Grp. B & Grp. C & Grp. D & & \\
& X \pm S.D & X \pm S.D & X \pm S.D & X \pm S.D & F- value & p \\
\hline PI & $6.00 \pm 2.35$ & $6.50 \pm 1.95$ & $7.07 \pm 1.69$ & $6.08 \pm 2.07$ & 0.79 & 0.50 \\
RM & $6.23 \pm 3.24$ & $6.29 \pm 3.00$ & $7.29 \pm 2.75$ & $6.42 \pm 3.60$ & 0.44 & 0.73 \\
LF $(\mathrm{cm})$ & $6.15 \pm 2.21$ & $6.36 \pm 1.39$ & $6.18 \pm 1.15$ & $6.23 \pm 0.71$ & 0.13 & 0.94 \\
SE & $38.50 \pm 5.55$ & $36.71 \pm 6.66$ & $37.14 \pm 8.73$ & $43.75 \pm 9.78$ & 2.17 & 0.10 \\
\hline
\end{tabular}

Key: $\mathrm{PI}=$ Pain intensity

$\mathrm{p}=$ probability at $0.05 \alpha$-level

$\mathrm{RM}=$ Activities limitation as measured by Roland Morris Disability Questionnaire

$\mathrm{X}=$ mean

LF $=$ Lumbar flexibility

$\mathrm{SE}=$ Self esteem

Grp A = Endurance exercise plus McKenzie Exercise plus Back education group

Grp B = Endurance exercise plus Back education group

Grp C $=$ McKenzie Exercise plus Back education group

Grp D $=$ Back education group

Table 2. ANOVA for comparison of mean change of participants' parameters from week 0 to 4 of the study

\begin{tabular}{lcccccc}
\hline $\mathrm{N}=53$ & Grp. A & Grp. B & Grp. C & Grp. D & & \\
& X \pm S.D & X \pm S.D & X \pm S.D & X \pm S.D & F- value & $p$ \\
\hline PI & $1.92 \pm 1.66$ & $1.86 \pm 1.70$ & $2.21 \pm 0.70$ & $0.58 \pm 0.90$ & 3.73 & $0.02^{*}$ \\
RM & $0.92 \pm 1.12$ & $1.64 \pm 1.82$ & $1.14 \pm 1.51$ & $0.50 \pm 1.45$ & 1.30 & 0.28 \\
LF $(\mathrm{cm})$ & $0.38 \pm 1.77$ & $0.04 \pm 0.11$ & $0.07 \pm 0.20$ & $0.02 \pm 0.04$ & 2.76 & $0.05^{*}$ \\
SE & $2.08 \pm 4.70$ & $0.50 \pm 1.16$ & $0.07 \pm 2.75$ & $2.08 \pm 3.32$ & 1.42 & 0.25 \\
\hline
\end{tabular}

Key: $F=$ ANOVA value

$\mathrm{PI}=$ Pain intensity

$\mathrm{p}=$ Probability at 0.05 alpha level

$\mathrm{RM}=$ Activities limitation as measured by Roland Morris Disability Questionnaire

$\mathrm{X}=$ Mean change

$\mathrm{LF}=$ Lumbar flexibility

$\mathrm{SE}=$ Self esteem

Grp A = Endurance exercise plus McKenzie Exercise plus Back education group

Grp B = Endurance exercise plus Back education group

Grp C $=$ McKenzie Exercise plus Back education group

Grp D= Back education group

Table 3. Post hoc analysis of participants' parameters at the end of the $4^{\text {th }}$ week of the study

\begin{tabular}{lllll}
\hline $\begin{array}{l}\text { N= 53 } \\
\text { GROUPS }\end{array}$ & M.D & Sig. & M.D & Sig. \\
\hline B v A & 0.07 & 1.00 & 0.32 & $0.03^{*}$ \\
C v A & 0.29 & 0.99 & 0.41 & $0.02^{*}$ \\
D v A & 1.34 & 0.11 & 0.37 & $0.03^{*}$ \\
C v B & 0.36 & 0.97 & 0.09 & 0.85 \\
D v B & 1.27 & 0.13 & 0.05 & 0.90 \\
D v C & 1.63 & $0.00^{*}$ & 0.04 & 0.95 \\
\hline
\end{tabular}

Key: PI = Pain intensity

$\mathrm{LF}=$ Lumbar flexibility

$\mathrm{MD}=$ Mean difference

Sig. $=$ Significance level

$*=$ Significant difference at $0.05 \alpha$-level parameters from week 0 to 8 of the study

At the end of week 8 of the study results obtained showed that there were significant differences across groups in pain intensity and activities limitation of participants $(\mathrm{p}<0.05)$ (Table 4$)$. Post hoc analysis showed that participants in group $\mathrm{C}$ had significantly higher reduction in pain intensity and $\mathrm{D}$, least reduction (Table 5).

Participants in group B had significantly greater reduction in activities limitation than those in the groups $\mathrm{A}$ and $\mathrm{C}$ at the end of the $8^{\text {th }}$ week of the study. Participants in group B had significantly higher reduction in activities limitation than those in the other two groups (Table 5). Hence at the end of the study, groups A, B and C except group D had significant reduction in activities limitation.

It was observed in this study that there was no significant difference in the lumbar flexibility of 
Table 4. ANOVA for comparison of mean change of participants' parameters from week 0 to 8 of the study

\begin{tabular}{lcccccc}
\hline $\mathrm{N}=53$ & Grp. A & Grp. B & Grp. C & Grp. D & & \\
& X \pm S.D & X \pm S.D & X \pm S.D & X \pm S.D & F- value & p \\
\hline PI & $4.62 \pm 2.50$ & $4.29 \pm 2.02$ & $5.86 \pm 1.66$ & $2.44 \pm 2.15$ & 5.91 & $0.00^{*}$ \\
RM & $5.00 \pm 3.00$ & $5.43 \pm 3.08$ & $4.64 \pm 1.74$ & $0.92 \pm 1.51$ & 8.90 & $0.00^{*}$ \\
LF (cm) & $0.40 \pm 1.03$ & $0.08 \pm 0.20$ & $0.04 \pm 1.22$ & $0.07 \pm 0.02$ & 0.88 & 0.46 \\
SE & $2.31 \pm 4.82$ & $1.14 \pm 4.09$ & $0.21 \pm 2.52$ & $3.83 \pm 4.49$ & 2.35 & 0.08 \\
\hline
\end{tabular}

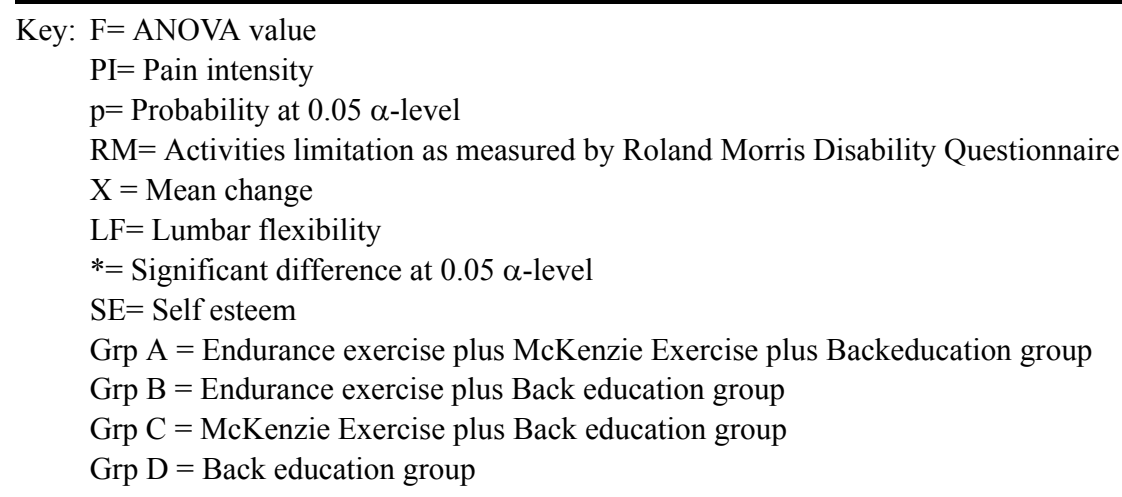

Table 5. Post hoc analysis of participants' parameters at the end of the $8^{\text {th }}$ week of the study

\begin{tabular}{lllll}
\hline N=53 & \multicolumn{2}{c}{ PI } & \multicolumn{2}{c}{ RM } \\
GROUPS & M.D & Sig. & M.D & Sig. \\
\hline B v A & 1.14 & $0.02^{*}$ & 0.37 & 0.61 \\
C v A & 0.14 & 0.77 & 1.41 & 0.06 \\
D v A & 2.59 & $0.00^{*}$ & 4.27 & $0.00^{*}$ \\
C v B & 1.00 & $0.03^{*}$ & 0.71 & $0.01^{*}$ \\
D v B & 1.45 & $0.00^{*}$ & 4.64 & $0.00^{*}$ \\
D v C & 2.45 & $0.00^{*}$ & 2.86 & $0.00^{*}$ \\
\hline
\end{tabular}

Key: PI = Pain intensity

$\mathrm{RM}=$ Activities limitation as measured by Roland Morris Disability Questionnaire

$\mathrm{N}=$ Total number of subjects

$\mathrm{MD}=$ Mean difference

Sig. $=$ Significance level

$*=$ Significant difference at $0.05 \alpha$-level

participants in the 4 groups at the end of week 8 of the study. This is understandable since the few participants $(28.3 \%)$ who could not touch their toes at week 0 as demonstrated during range of motion measurement with the modified Schober technique were able to do so by end of the $4^{\text {th }}$ week of the study. No further improvement in lumbar flexibility could hence be expected since touching of toes is the end point of lumbar flexion when it is measured using the same modified Schober technique. It was also observed in this study that there was no significant difference in the self esteem of participants in the 4 groups at the end of the study.

\section{Discussion}

Participants' profile

A $27.4 \%$ attrition rate was observed in this study. This observation might be linked with the observation that patients in this environment usually stop coming for treatment once they think they are well enough and not necessarily when they have completed a study they consented to participate in.

Torstensten ${ }^{34)}$; Rittweger et al. ${ }^{35)}$, in their studies on long-term LBP among non-Nigerians reported a $15 \%$ attrition rate. While Akosile et al. ${ }^{36)}$ in a study involving Nigerians with LBP reported $30 \%$ attrition.

The observation that $60.4 \%$ of the participants were females suggests a higher prevalence of LBP in female Nigerians. Waddell ${ }^{37)}$; Tomas et al. ${ }^{38)}$; Aclouffe et al. ${ }^{39)}$ have similarly reported a higher prevalence of low back pain in women and a preponderance of risk factors for low back pain in females, while Helliovara ${ }^{40)}$ and Wing ${ }^{41)}$, reported that LBP is equally common in men and women. This was however a hospital based study and not a prevalence study. The average age of participants in this study was $45.3 \pm 8.1$ years. This observation is in line with that of Wing ${ }^{41)}$ who opined that low back pain occurs usually in the thirties to fifties and also in older women. Also Bio et al. ${ }^{42}$, in a prevalence study reported the mean age for the occurrence of LBP to be $40 \pm 5.6$ years.

\section{Across group comparison of mean change of participants' parameters from week 0 to 4 of the study}

The observation that there was reduction in pain intensity and improvement in flexibility is similar to that of Kofotolis and Eleftherios ${ }^{43)}$, who reported improvement in 
pain and lumbar mobility after 4 weeks of variety of exercises in treatment of LBP. In this present study post hoc analysis showed that group A had significantly higher increase in lumbar flexibility than the other groups. This may be because group A had both endurance and McKenzie exercises, and exercising the back improves back flexibility by keeping the connective fibers of ligaments and tendons flexible ${ }^{44,45)}$.

\section{Across group comparison of mean change of participants' parameters from week 0 to 8 of the study}

There was significant reduction on pain intensity in all the groups studied. Exercises of various types have been found in literature to be effective in the management of long-term $\mathrm{LBP}^{1,25,46,)}$, and Oleske et al. ${ }^{47)}$ opined that back care education can be used in the management of LBP without any additional physiotherapy procedures.

The observation that group $\mathrm{C}$ had significantly higher pain reduction than the other three groups is probable because McKenzie exercise is known as a pain modulator albeit in managing acute $\left.\mathrm{LBP}^{25}, 48,49\right)$; this present study however involved individuals with long-term LBP. Petersen et al ${ }^{50)}$ however treated individuals with sub-acute and long-term LBP using McKenzie exercise and reported McKenzie exercise to be as effective as strength training exercises with resultant significantly higher reduction in pain intensity in the McKenzie group.

Regimens that included exercises (A, B and C) effected higher significant improvement in activities limitation than the back care regimen (D). This might be because group D participants were not treated with exercises. Exercises effect gain in muscle strength (force-generating capacity), endurance and flexibility, restore injured tissues, and contributes to the ability of the back to sustain normal daily activities ${ }^{51)}$. Moffet et al. ${ }^{52)}$ reported similarly in their randomised controlled trial involving individuals with LBP that those in the exercise classes were able to function better according to Roland Morris disability questionnaire scores than participants in the control group who received no exercises. Endurance exercise for back muscles reconditions the back ${ }^{53,54)}$ and subsequently improves activities limitation observed among patients with LBP consequent to decreased levels of muscular endurance of the lumbar extensors and abdominal muscles ${ }^{6}$. Back care education on the other hand has generally been accepted as only an important adjunct to other physiotherapy procedures in the management of low back pain and not necessarily as an effective means of managing LBP ${ }^{55-57)}$, though Oleske et al. $^{47)}$ have opined that back care education can be used solely in the management of low back pain.

Exercise has been found to improve self-confidence and self-esteem ${ }^{58,59)}$, but the observation is contrary in this study. There was $27.4 \%$ attrition in this study and this would have affected the outcome of this study with respect to its external validity. A further study in this area is therefore recommended to improve on this.

Regimens A, B and C were effective in treating longterm LBP and in view of the fact that regimen $\mathrm{A}$ combines the components of regimens $\mathrm{B}$ and $\mathrm{C}$, regimen $\mathrm{A}$ is preferably recommended in the treatment of long-term mechanical LBP.

\section{References}

1) Shiple BJ: Treating low-back pain. Exercise knowns and unknowns. Phys Sports Med. 1997, 25: 51-66.

2) Nwuga G, Nwuga V: Relative therapeutic efficacy of the Williams and McKenzie protocols in back pain management. Phy Prac. 1985, 1: 99-105.

3) McKenzie RA: The Lumbar Spine: Mechanical Diagnosis and Therapy Spinal Publication. Waikanae; New Zealand, Spinal Publication Limited. 8 1981, P120.

4) Waddell G: A new clinical model for the treatment of low back pain. Spine. 1987, 12: 632-644.

5) de Vries H: EMG fatigue curve in postural muscles. A possible aetiology for idiopathic low back pain. Am J Phys Med. 1968, 47: 182-191.

6) Biering-Sorenson F: Physical measurements as risk indicators for low back trouble over a one-year period. Spine. 1984, 9: 106-119.

7) Foster $\mathrm{DN}$, Fulton $\mathrm{MN}$ : Back pain and the exercise prescription. Clin Sports Med. 1991, 10: 187-209.

8) McGill SM: Low back exercises: Evidence for improving exercise regimens. Phys Ther. 1998, 78: 754-765.

9) Mannion AF, Muntener M, Taimela S, Dvorak J: Comparison of three active therapies for low back pain: result of a randomized clinical trial with one-year follow-up. Rheumatology. 2001, 40: 772-778.

10) Nourbakhsh MR, Arab AM: Relationship between mechanical factors and incidence of low back pain. J Orthop Sports Phys Ther. 2002, 32: 447-460.

11) Adegoke BOA, Babatunde FO: Effect of an exercise protocol on the endurance of trunk extensor muscles: a RCT. HKPA Journal. 2007, 25: 2-9.

12) Omokhodion FO: Low back pain in a rural community in South West Nigeria. W Afr J Med, 2002, 21: 87-90.

13) Hazard RG: Chronic low back pain and disability. The efficacy of functional restoration. Bull Hosp Joint Dis. 1996, 55: 213 216.

14) Louw QA, Morris LD, Grimmer-Somers K: The prevalence of low back pain in Africa: a systematic review. BMC Musculoskelet Disord. 2007, 8: 105.

15) Ehrlich GE: Low Back Pain. Bull World Health Organ. 2003, 81:9.

16) Frank JW, Kerr MS, Broker AS: Disability resulting from occupational low back pain, part 1 what do we know about primary prevention? A review of the scientific evidence on prevention before disability begins. Spine. 1996, 21: 29082917.

17) Volinn E: The epidemiology of low back pain in the rest of the world. A review of surveys in low- and middle-income countries. Spine. 1997, 22: 1747-1754.

18) Walker BF: The prevalence of low back pain: a systematic 
review of the literature from 1966 to 1998. J Spinal Disord. 2000, 13: 205-217.

19) Jenkins EM, Borenstein DG: Exercise for low back patient. Ballieres Clin Rheumatol. 1994, 8: 191-197.

20) Moffat KJ, Richardson C, Sheldon T: Back Pain; its management and cost to society. Discussion paper 129. New York University Center for Health Economics; USA, 1995.

21) Feurstein M, Beattie P: Behavioral factors affecting pain and disability in low back pain: mechanism and assessment. Phys Ther. 1995, 75: 267-280.

22) Hayden J, van Tulder M, Malmivaara A, Koes B: Metaanalysis: exercise therapy for non-specific low back pain. Ann Intern Med. 2005, 142: 765-775

23) Samanta J, Kendall J, Samanta A: 10-minute consultation: chronic low back pain. BMJ. 2003, 326: 535.

24) Bigos SJ, Bowyer OR, Braen GR: Acute Low Back Problems in Adults: Clinical Practice Guideline Number 14. Agency for Health Care Policy and Research Publication, 95643, Rock Ville, MD, Public Health Service, US Department of Health and Human Services. 1994.

25) van Tulder MW, Touray T, Furlan AD, Bombardier C, Bouter L: Editorial board of the cochrane collaborators updated method guidelines for systematic reviews in the cochrane collaboration back review. Spine. 2003, 28: 1290-1299.

26) Verrills $P$, Vivian D: Interventions in chronic low back pain. Aust Fam Physician. 2004, 33: 421-426.

27) Merrit J, Mc Lean T, Erickson R, Offord K: Measurement of trunk flexibility in normal subjects: reproducibility of three clinic methods. Mayo Clinic Production. 1986, 61: 192-197.

28) Chapman-Smith D: Measuring results - the new importance of patient questionnaires. Chiro Report. 1992, 7: 1-6.

29) Fairbank JC, Davies JB, Couper J, O'Brien JP: The Oswestry low back pain disability questionnaire. Physiotherapy. 1980, 66: 271-273.

30) Adanijo IB, Oyefeso AO: Developing a Self Report Scale of Self Esteem: A Paper presented at the $3^{\text {rd }}$ Annual Conference of the Nigerian Psychological Association, Nzukka, 1986.

31) http://www.chirogeek.com/003_Exercise_Main_Page.htm (Accessed 13 October, 2005)

32) Mckenzie RA: Treat Your Own Back. Spinal Publication. Waikanae; New Zealand, Spinal Publication Limited, 1990, PP37-48.

33) Odebiyi DO: How to avoid back pain at work and rest. A Handbook of the Nigerian Back School. Ibadan, Crowntex Printing Press, 2004, PP4-13.

34) Torstensen TA, Ljunggren AE, Meen HD: Efficiency and costs of medical exercise therapy, conventional physiotherapy, and self-exercise in patients with chronic low back pain: a pragmatic, randomized, single-blinded, controlled trial with 1year follow-up. Spine. 1998, 23: 2616-2624.

35) Rittweger J, Just K, Kautzsch K, Reeg P, Felsenberg D: Treatment of chronic lower back pain with lumbar extension and whole-body vibration exercise. Spine. 2002, 27: 18291834.

36) Akosile CO, Nwankwo EI, Johnson OE, Raji FS: Comparative effect of different Mckenzie extension exercise-based protocols on spinal flexibility of low back pain patients. J ICHPER. 2006, 1: 14-18.

37) Waddell G: CSAG report on back pain. London: HMS. The epidemiology of back pain. CSAG Report. 1994, 1-64.

38) Reigo T, Timpka T, Tropp H: The epidemiology of back pain in vocational age groups. Scand J Prim Health Care. 1999, 17: $17-21$.

39) Alcouffe J, Maniller P, Brehier M, Fabin C, Faupin F: Analysis by sex of low back pain among workers from small companies in the paris area: severity and occupational consequences. OEM. 1999, 10: 696-701.

40) Helliovaara M: Risk factors for low back pain and sciatica. Ann Med. 1989, 21: 257-264.

41) Wing PC: Minimizing disability in patients with low-back pain. CMAJ. 2001, 16: 1459.

42) Bio FY, Sadra S, Jackson C, Burge PS: Low back pain in underground gold miners in Ghana. GMJ. 2007, 14: 21-25.

43) Kofotolis N, Kellis E: Effects of two 4-week proprioceptive neuromuscular facilitation programs on muscle endurance, flexibility, and functional performance in women with chronic low back pain. Phys Ther. 2006, 86: 1001-1012.

44) Mayer T, Tabor J, Bovasso E, Gatchel E: Physical progress and residual impairment quantification after functional restoration. Part II: Lumbar mobility. Spine. 1994, 19: 389-394.

45) Karan A, Muslumanoglu L, Aki S, Filiz M, Yildiz E, Berker E: The comparison of the efficacy aerobic exercises versus strengthening, stretching and mobilization exercises in subacute and chronic LBP. Trop J Med Sci. 1999, 29: 475-479.

46) Manniche C, Hesselsoe G, Bentzen L, Christensen I, Lundberg E: Clinical trial of intensive muscle training for chronic low back pain. Lancet. 1988, ii: 1473-1476.

47) Oleske DM, Lavender SA, Andersson GB, Kwasny MM: Are back supports plus education more effective than education alone in promoting recovery from LBP? Results from a randomised controlled trial. Spine. 2007, 32: 2050-2057.

48) Clare HA, Adams RA, Maher CG: A systematic review of efficacy of McKenzie therapy for spinal pain. Aust J Physiother. 2004, 5: 209-216.

49) Machado LA, de Souza MS, Ferreira PH, Ferreira ML: The McKenzie method for low back pain: a systematic review of the literature with a meta-analysis approach. Spine. 2006, 31 : 254-262.

50) Petersen T, Kryger P, Ekdahl C, Olsen S, Jacobsen J: The effects of Mckenzie therapy as compared with that of intensive strengthening training for the treatment of patients with sub acute or chronic low back pain. Spine. 2002, 15: 172-179.

51) Malkia E, Ljunggren AE: Exercise programs for subjects with low back disorders. Scand J Med Sc Sports. 1996, 6: 73-81.

52) Moffett JK, Torgerson D, Bell Syer S, Jackson D, Llewlyn Phillips H, Farrin A, Barber J: Randomised controlled trial of exercise for low back pain: clinical outcomes, costs, and preferences. BMJ. 1999, 319: 279-283.

53) Johannes F, Remvig L, Kryger P, Beck P, Warming S, Lybeck $\mathrm{K}$, Dreyer V, Larsen LH: Exercise for chronic low back pain: a clinical trial. J Orthop Sports Phys Ther. 1995, 22: 52-59.

54) Carpenter DM, Nelson BW: Low back strategies for prevention and treatment of low back pain. Med Sci Sports Exerc. 1999, 31: 18-24.

55) Lønn JH, Glomsrød B, Soukup MG, Bø K, Larsen S: Active back school: prophylactic management for low back pain: a randomized controlled 1-year follow-up study. Spine. 1999, 24: $865-871$. 
56) Bendix AF, Bendix T, Lund C, Kirkbak S, Ostenfeld S: Comparison of three intensive programs for chronic low back patients: a prospective observer-blinded study with one year follow-up. Scand J Rehabil Med. 1997, 29: 81-89.

57) Daltroy LH, Iversen MD, Larson MG, Ryan J, Zwerling C, Fossel AH, Liang MH: A controlled trial of an educational program to prevent low back injuries. NEJM. 1997, 337: 322 328.

58) Folkins $\mathrm{CH}$, Sime WE: Physical fitness training and mental health. Am Psychol. 1981, 36: 373-389.

59) Geffen SJ: Rehabilitation principles for treating chronic musculoskeletal injuries. Med J Austr. 2003, 178: 238-242. 